EPJ Web of Conferences 66, 11009 (2014)

DOI: $10.1051 /$ epjconf/ 20146611009

(C) Owned by the authors, published by EDP Sciences, 2014

\title{
Research and development on materials for the SPES target
}

\author{
Stefano Corradetti ${ }^{1, a}$, Alberto Andrighetto ${ }^{1}$, Mattia Manzolaro ${ }^{1}$, Daniele Scarpa ${ }^{1}$, Jesus \\ Vasquez $^{1,2}$, Massimo Rossignoli ${ }^{1}$, Alberto Monetti ${ }^{1}$, Michele Calderolla ${ }^{1}$, and Gianfranco Prete ${ }^{1}$ \\ ${ }^{1}$ INFN - Laboratori Nazionali di Legnaro, Viale dell'Universitá 2, 35020 Legnaro (PD), Italy \\ ${ }^{2}$ Universitá di Padova, Dipartimento di Ingegneria dell'Informazione, Via Gradenigo 6/B, 35131 Padova, Italy
}

\begin{abstract}
The SPES project at INFN-LNL (Istituto Nazionale di Fisica Nucleare - Laboratori Nazionali di Legnaro) is focused on the production of radioactive ion beams. The core of the SPES facility is constituted by the target, which will be irradiated with a 40 $\mathrm{MeV}, 200 \mu \mathrm{A}$ proton beam in order to produce radioactive species. In order to efficiently produce and release isotopes, the material constituting the target should be able to work under extreme conditions (high vacuum and temperatures up to $2000^{\circ} \mathrm{C}$ ). Both neutronrich and proton-rich isotopes will be produced; in the first case, carbon dispersed uranium carbide $\left(\mathrm{UC}_{x}\right)$ will be used as a target, whereas to produce p-rich isotopes, several types of targets will have to be irradiated. The synthesis and characterization of different types of material will be reported. Moreover, the results of irradiation and isotopes release tests on different uranium carbide target prototypes will be discussed.
\end{abstract}

\section{Introduction}

The aim of the SPES (Selective Production of Exotic Species) project, now under construction at INFN-LNL (Istituto Nazionale di Fisica - Laboratori Nazionali di Legnaro) is the development of a facility for the production of radioactive ion beams (RIBs) to be delivered to experimental users for nuclear physics, astrophysics and materials science studies [1]. SPES is designed to be an ISOL (Isotope Separation On-Line) facility, in which the radioactive isotopes of interest are produced by the direct interaction of a primary beam with a suitable target, and directly extracted and ionized to be accelerated and delivered to specific experimental areas. In the case of SPES, the primary beam is constituted by $40 \mathrm{MeV}$ protons, provided by a cyclotron, with scheduled operational intensity of 200 $\mu \mathrm{A}$. Once produced by different nuclear reactions involving the primary beam and the target nuclei (mainly fission), the isotopes are rapidly extracted from the target by keeping it at high temperature in high vacuum $\left(2000^{\circ} \mathrm{C}, 10^{-6} \mathrm{mbar}\right)$ and ionized by means of one of the following processes, depending on the isotopes properties: surface ionization (very effective for low ionization potential elements such as alkali or alkaline-earth metals), laser-induced ionization (highly selective for a wide range of elements) or electron-impact ionization (plasma ion source, capable of ionizing high ionization potential elements, but not very selective). The obtained ions are then extracted by means of a $30 \mathrm{kV}$ potential, mass separated and re-accelerated in the ALPI superconducting LINAC with energies of 10 $\mathrm{AMeV}$ and higher.

a e-mail: stefano.corradetti@lnl.infn.it

This is an Open Access article distributed under the terms of the Creative Commons Attribution License 2.0, which permits unrestricted use, distribution, and reproduction in any medium, provided the original work is properly cited. 


\section{The SPES target}

The target represents the core of the SPES project, and therefore a huge research and development program is currently being carried out in order to design and test materials capable to efficiently produce and release the highest possible amount of radioactive ions. The SPES target layout is innovative if compared to that of other facilities since it is not composed of a unique block of refractory material, but it instead consists of seven separate thin discs, as shown in fig. 1. This particular layout has the aim of efficiently dissipate the $8 \mathrm{~kW}$ total power deposited on the discs during the irradiation [1], thus guaranteeing their structural integrity over the whole RIBs production period. The properties required for the SPES target material are strictly correlated to the enhancement of the processes undergoing between the isotopes production and their release, which can be divided into two stages:

- The diffusion of the isotopes, generated inside the material grains, towards the surface.

- The effusion in high vacuum, either in the material pores or in the free spaces surrounding the target discs, towards the ion source.

The obtainment of a fast release of isotopes depends on many parameters, relative to the target material and to the operative conditions. The properties required for a material to work with a high efficiency as an ISOL target can be summarized as [2]:

- It must be able to work for a sufficient amount of time at high temperatures (more than $2000{ }^{\circ} \mathrm{C}$ ) in high vacuum without degradation of its properties, in order to speed up the diffusion-effusion mechanism.

- It should exhibit high thermal emissivity and conductivity in order to dissipate the power generated by its interaction with the primary beam without undergoing structural damage.

- It should be highly permeable to exotic species during their path towards the ion source. This behavior is strictly related to the presence of open interconnected porosity, which favors the escape of species from the target [3].

\section{Materials for the SPES target}

The research on materials for the SPES target, as in the case of other existing or future ISOL facilities [3], represents an important part of the entire facility development, for the reasons described in section

2. The following paragraphs report the main properties of the two main classes of materials produced and characterized by the SPES target group.

\subsection{Uranium carbide: production of $n$-rich isotopes}

The most used ISOL target material is uranium carbide [2], since it not only possesses the requisites highlighted in section 2, but is also able to generate, by fission reactions, a wide spectrum of elements when interacting with a proton beam. The most common type of uranium carbide produced, tested and used in ISOL facilities can be referred to as $\mathrm{UC}_{x}$, indicating it is composed of uranium dicarbide $\left(\mathrm{UC}_{2}\right)$, graphite $(\mathrm{C})$, and a minor amount of uranium monocarbide (UC). In recent years, the synthesis of uranium carbide thin discs have been carried out $[2,4]$, by using the reaction:

$$
\mathrm{UO}_{2}+6 \mathrm{C} \rightarrow \mathrm{UC}_{2}+2 \mathrm{C}+2 \mathrm{CO}
$$

which was made to occur at high temperature (up to $1800^{\circ} \mathrm{C}$ ) in high vacuum. Particular attention was given to the choice of the uranium and carbon sources of reaction (1), studying their effect in the 


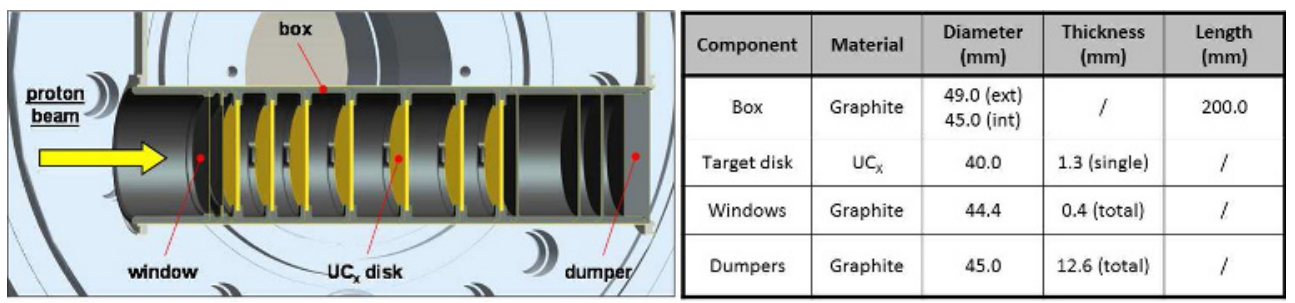

Figure 1. Schematic representation of the SPES target, with indication of the main components dimensions.

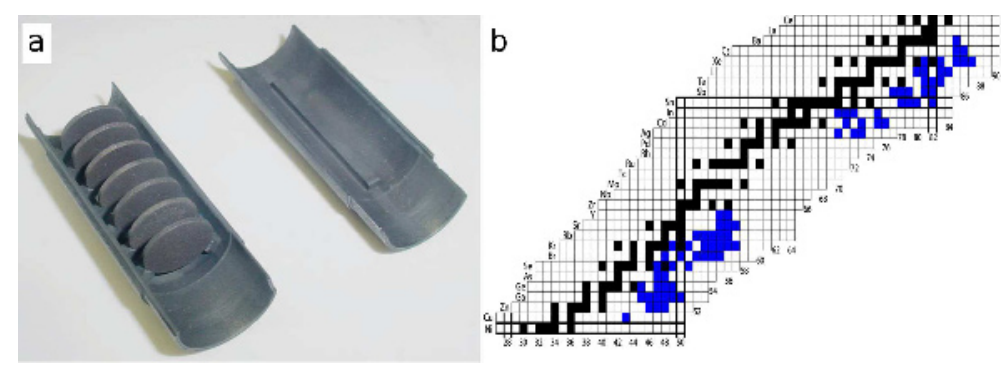

Figure 2. a: a $\mathrm{UC}_{x}$ target prototype used for irradiation tests, b: isotopes released from two different $\mathrm{UC}_{x}$ targets.

final material properties, in terms of composition and microstructure. After off-line characterization, two target prototypes, constituted by seven $\mathrm{UC}_{x}$ thin discs, with the same layout of fig. 1 but with scaled dimensions (13 mm diameter, as shown in fig. 2a) were tested at the OLTF (On-Line Test Facility) of Oak Ridge National Laboratory (ORNL). The two materials differed in the carbon source used in reaction (1): in the first case, graphite was used, whereas in the second material a graphite carbon nanotube mixture was used, resulting in a higher porosity of the final material.

The on-line irradiation tests performed at OLTF with a $50 \mathrm{nA}, 40 \mathrm{MeV}$ primary proton beam highlighted the capability of the two different materials to produce and release a set of more than 75 isotopes, with half-lives ranging from seconds up to few hours [4, 5], as reported schematically in fig. $2 \mathrm{~b}$. Future activities will involve the synthesis and test of $\mathrm{UC}_{x}$ prototypes of the real SPES target dimensions (40 mm diameter).

\subsection{Other materials}

Besides uranium carbide, several other materials can be irradiated in ISOL facilities to produce different nuclei, typically in the neutron-deficient (p-rich) side of the stable isotopes. In other cases, the synthesis and characterization of a material can serve as a "test" to develop and improve techniques for the production of other ISOL target materials, as in the case of lanthanum carbide described below. The following paragraph presents three examples of materials development for the SPES target.

Silicon carbide will be among the first irradiated materials at SPES, in order to produce p-rich aluminum isotopes. A SPES target prototype composed of seven thin SiC discs has been already tested at OLTF, proving the possibility to obtain ${ }^{26} \mathrm{Al}$ from it [6].

Porous boron carbide has been proposed recently as a target for the production of p-rich Be beams, in particular ${ }^{7} \mathrm{Be}$, to be used for astrophysics experiments. Research carried out at SPES demonstrated 


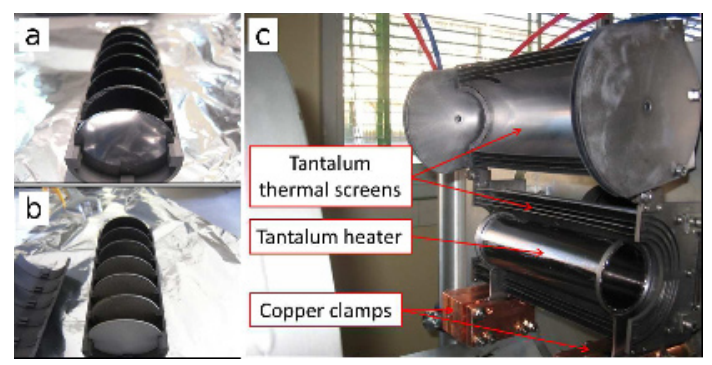

Figure 3. a: seven $\mathrm{La}_{2} \mathrm{O}_{3}+\mathrm{C}$ discs before the thermal treatment, contained in a graphite box. b: lanthanum carbide discs after the thermal treatment. c: main components of the furnace: the box containing the discs is inserted inside the tantalum heater, which is heated by Joule effect and surrounded by tantalum screens.

the possibility to obtain porous $\mathrm{B}_{4} \mathrm{C}$ based discs by means of two different techniques, both based on the use of boric acid as a boron source, reacting it with proper carbon sources [7].

Lanthanum carbide has been extensively used in past years [8] as a test material to develop devices and techniques for the obtainment of carbide targets by means of reaction:

$$
\mathrm{La}_{2} \mathrm{O}_{3}+11 \mathrm{C} \rightarrow 2 \mathrm{LaC}_{2}+4 \mathrm{C}+3 \mathrm{CO}
$$

Recently, the attention has been focused on the development of a device in which to perform reaction (2) for an entire target in a single run. The furnace allows for the reaction of seven $\left(\mathrm{La}_{2} \mathrm{O}_{3}+11 \mathrm{C}\right)$ discs up to $1800{ }^{\circ} \mathrm{C}$ inside a graphite box (see fig. 3a), which is inserted in a tantalum heater, kept at high temperature inside a vacuum chamber. Figure 3 shows a graphite box containing seven La-based samples before $\left(\mathrm{La}_{2} \mathrm{O}_{3}+\right.$ graphite $)$ and after $\left(\mathrm{LaC}_{x}\right)$ reaction, together with a view of the furnace main components. Due to the similarity between $\mathrm{LaC}_{x}$ and $\mathrm{UC}_{x}$ properties and synthesis, the device here described can be applied to single-run production of $\mathrm{UC}_{x}$ targets; in this way, after the treatment, the box containing the carbide discs can be transferred inside the irradiation chamber without handling separately each $\mathrm{UC}_{x}$ pellet.

\section{Conclusions}

The research and development of materials is a key activity towards the SPES facility construction and operation. Several materials have been proposed and tested to study their properties and their capability to produce and release radioactive isotopes, in particular uranium carbide. Other materials are as well considered as potential SPES targets.

\section{References}

[1] A. Andrighetto et al., AIP Conf. Proc. 1491, 58 (2012).

[2] L. Biasetto et al., J. Nucl. Mater. 404, 68 (2010).

[3] B. Hy et al., Nucl. Instrum. Methods Phys. Res. B 288, 34 (2012).

[4] S. Corradetti et al., Eur. Phys. J. A 49, 56 (2013).

[5] S. Corradetti et al., Eur. Phys. J. A 47, 119 (2011).

[6] M. Barbui et al., Nucl. Instrum. Methods Phys. Res. B 266, 4289 (2008).

[7] S. Corradetti et al., J. Nucl. Mater. 432, 212 (2013).

[8] L. Biasetto et al., J. Nucl. Mater. 378, 180 (2008). 